\title{
Erratum to: Two-tailed approximate confidence intervals for the ratio of proportions
}

\author{
A. Martín Andrés ${ }^{1}$ • M. Álvarez Hernández ${ }^{1}$
}

Published online: 18 December 2015

(C) Springer Science+Business Media New York 2015

\section{Erratum to: Stat Comput (2014) 24:65-75 DOI 10.1007/s11222-012-9353-5}

The authors apologize for the errors made in the article which appeared in Statistics and Computing (2014) 24:65-75. Some of these are of slight importance, but one error is quite substantial.

The less important errors are:

- Page 65, left column, line 11: where it says “... (3) the best methods among ..." it should say "...(3) the best method among ...".

- Page 73, left column, line 11: where it says “... the expression (19) ..." it should say “... the expression (18) ...".

- Page 74, left column, line 25: where it says “... working out $p_{i E}$ one gets ..." it should say “... working out $p_{1 E}$ one gets ...".

But the most outstanding error is in Sect. 4, comprising all the text between expressions (15) and (20) inclusive, which occurs when the explicit formula of the CI about the relative risk is being deduced from the optimal method ZA1. The error originates in the fact that the estimators for the unknown proportions $p_{i}$ used in the said method are $p_{1 A}=$ $\operatorname{Min}\left\{1 ;\left(x_{2}+x_{1} \rho\right) / n \rho\right\}$ and $p_{2 A}=\operatorname{Min}\left\{1 ;\left(x_{2}+x_{1} \rho\right) / n\right\}$,

The online version of the original article can be found under doi:10.1007/s11222-012-9353-5.

\section{A. Martín Andrés} amartina@ugr.es

1 Department of Biostatistics, Faculty of Medicine, University of Granada, 18071 Granada, Spain as is indicated in expression (12) of the paper. However the estimators in expression (15) of the paper do not correspond to the first ones: they correspond to another version of these that yields a method that behaves a good deal more badly. As a result, all the text between expressions (15) and (20) inclusive in the paper should be replaced by the text that appended here (below). It can be seen that the new first expressions in (15), (16) and (17), as well as the new expression (18), are the same as those in the paper, except for an error that is contained in the expression (17) in the paper. The replacement text is:

$$
\left\{\begin{array}{lll}
p_{1 A}=\frac{x_{2}+\rho x_{1}}{n \rho}, & p_{2 A}=\frac{x_{2}+\rho x_{1}}{n} & \text { if } \rho_{1}=\frac{x_{2}}{n-x_{1}} \leq \rho \leq \rho_{2}=\frac{n-x_{2}}{x_{1}} \\
p_{1 A}=1, & p_{2 A}=\frac{x_{2}+\rho x_{1}}{n} & \text { if } \rho<\rho_{1}=\frac{x_{2}}{n-x_{1}} \\
p_{1 A}=\frac{x_{2}+\rho x_{1}}{n \rho}, & p_{2 A}=1 & \text { if } \rho>\rho_{2}=\frac{n-x_{2}}{x_{1}}
\end{array}\right.
$$

from which it can be deduced that the test statistic for procedure ZA will be:

$$
\begin{aligned}
z_{Z A}^{2} & =\frac{\left(\bar{p}_{2}-\rho \bar{p}_{1}\right)^{2}}{\rho^{2} \frac{p_{1 A} q_{1 A}}{n_{1}}+\frac{p_{2 A} q_{2 A}}{n_{2}}} \\
& = \begin{cases}\frac{n n_{1} n_{2}\left(\bar{p}_{2}-\rho \bar{p}_{1}\right)^{2}}{\left(x_{2}+\rho x_{1}\right)\left\{\left(n_{1}-x_{2}\right)+\rho\left(n_{2}-x_{1}\right)\right\}} & \text { if } \rho_{1} \leq \rho \leq \rho_{2} \\
\frac{n^{2} n_{2}\left(\bar{p}_{2}-\rho \bar{p}_{1}\right)^{2}}{\left(x_{2}+\rho x_{1}\right)\left\{\left(n-x_{2}\right)-\rho x_{1}\right\}} & \text { if } \rho<\rho_{1} \\
\frac{n^{2} n_{1}\left(\bar{p}_{2}-\rho \bar{p}_{1}\right)^{2}}{\left(x_{2}+\rho x_{1}\right)\left\{\left(n-x_{1}\right) \rho-x_{2}\right\}} & \text { if } \rho>\rho_{2} .\end{cases}
\end{aligned}
$$

Making $z_{Z A}^{2}=z_{\alpha / 2}^{2}$, the CI sought $\left(\rho_{L}, \rho_{U}\right)$ is given by the solutions to the equations: 


$$
\begin{cases}x_{1}\left\{n n_{2} \bar{p}_{1}-z_{\alpha / 2}^{2}\left(n_{2}-x_{1}\right)\right\} \rho^{2}-\left\{2 n x_{1} x_{2}+z_{\alpha / 2}^{2}\left(n_{1} x_{1}+n_{2} x_{2}-2 x_{1} x_{2}\right)\right\} \rho & \\ \quad+x_{2}\left\{n n_{1} \bar{p}_{2}-z_{\alpha / 2}^{2}\left(n_{1}-x_{2}\right)\right\}=0, & \text { if } \rho_{1} \leq \rho \leq \rho_{2} \\ \left(n^{2} n_{2} \bar{p}_{1}^{2}+z_{\alpha / 2}^{2} x_{1}^{2}\right) \rho^{2}-\left[2 n^{2} \bar{p}_{1} x_{2}+z_{\alpha / 2}^{2} x_{1}\left(n-2 x_{2}\right)\right] \rho & \\ \quad+x_{2}\left\{n^{2} \bar{p}_{2}-z_{\alpha / 2}^{2}\left(n-x_{2}\right)\right\}=0, & \text { if } \rho<\rho_{1} \\ x_{1}\left\{n^{2} \bar{p}_{1}-z_{\alpha / 2}^{2}\left(n-x_{1}\right)\right\} \rho^{2}-\left[2 n^{2} \bar{p}_{2} x_{1}+z_{\alpha / 2}^{2} x_{2}\left(n-2 x_{1}\right)\right] \rho & \\ \quad+\left(n^{2} n_{1} \bar{p}_{2}^{2}+z_{\alpha / 2}^{2} x_{2}^{2}\right)=0, & \text { if } \rho>\rho_{2},\end{cases}
$$

where $x_{i} \neq 0$ and $n-x_{i} \neq 0$ because the data have been increased by 0.5 . Hence the first step is to determine the two solutions for the first equation in expression (17):

$\left(\rho_{L}, \rho_{U}\right)=\frac{n x_{1} x_{2}+\frac{z_{\alpha / 2}^{2}\left(n_{1} x_{1}+n_{2} x_{2}-2 x_{1} x_{2}\right)}{2} \pm z_{\alpha / 2} \sqrt{n^{2} x_{1} x_{2}\left(a_{1}-n \bar{p}_{1} \bar{p}_{2}\right)+\left\{\frac{z_{\alpha / 2}\left(n_{2} x_{2}-n_{1} x_{1}\right)}{2}\right\}^{2}}}{x_{1}\left\{n n_{2} \bar{p}_{1}-z_{\alpha / 2}^{2}\left(n_{2}-x_{1}\right)\right\}}$

If the two solutions obtained $\left(\rho_{L}, \rho_{U}\right)$ verify that $\rho_{1} \leq \rho_{L}<$ $\bar{R}<\rho_{U} \leq \rho_{2}$, the problem is ended. Otherwise, one or two of the boundaries may fail. If the boundary that fails is $\rho_{L}$, one must obtain the smaller of the two solutions in the second equation of expression (17):

$$
\begin{aligned}
\rho_{L}= & \frac{1}{x_{1}\left\{\frac{n n_{2}}{n_{1}^{2}}+\frac{z_{\alpha / 2}^{2}}{n}\right\}}\left\{x_{2} \frac{n}{n_{1}}+z_{\alpha / 2}^{2}\left(\frac{1}{2}-\frac{x_{2}}{n}\right)\right. \\
& -z_{\alpha / 2} \sqrt{\left.\frac{n^{2}}{n_{1}^{2}} \frac{x_{2} y_{2}}{n_{2}}+\frac{z_{\alpha / 2}^{2}}{4}\right\} .}
\end{aligned}
$$

but if $\rho_{L}<0$, it should be $\rho_{L}=0$. If the boundary that fails is $\rho_{U}$, one must obtain the solution $\rho>\bar{R}$ in the third equation of expression (17):

$$
\begin{aligned}
\rho_{U}= & \frac{1}{\frac{x_{1}}{x_{2}}\left\{\frac{n x_{1}}{n_{1}}-\frac{z_{\alpha / 2}^{2}}{n}\left(n-x_{1}\right)\right\}}\left\{x_{1} \frac{n}{n_{2}}+z_{\alpha / 2}^{2}\left(\frac{1}{2}-\frac{x_{1}}{n}\right)\right. \\
& \left.+z_{\alpha / 2} \sqrt{\frac{n^{2}}{n_{2}^{2}} \frac{x_{1} y_{1}}{n_{1}}+\frac{z_{\alpha / 2}^{2}}{4}}\right\} .
\end{aligned}
$$

but if $\rho_{U}<\bar{R}$, it should be $\rho_{U}=+\infty$.

Acknowledgments This research was supported by the Spanish Ministerio de Economía y Competitividad, Grant Number MTM201235591 (cofinanced by funding from FEDER). 